\title{
EPIDEMIOLOGÍA DEL PSEUDOTUMOR CEREBRAL
}

\section{EPIDEMIOLOGY OF PSEUDOTUMOR CEREBRI}

\author{
ASENSIO-SÁNCHEZ VM ${ }^{1}$, MERINO-ANGULO J ${ }^{1}$, MARTÍNEZ-CALVO S ${ }^{2}$, CALVO MJ ${ }^{2}$, \\ RODRÍGUEZ R ${ }^{3}$
}

\section{RESUMEN}

Objetivo: Determinar la epidemiología del pseudotumor cerebral (PTC) en los pacientes de nuestro servicio de oftalmología.

Métodos: Se revisaron las historias clínicas de todos los pacientes diagnosticados de PTC en el hospital entre los años 1994 y 2004, considerándose para el estudio los PTC diagnosticados por el servicio de oftalmología. Se recogieron los datos del sexo, edad al diagnóstico, índice masa corporal (IMC), presión de salida del líquido cefalorraquídeo (LCR), y los resultados de la TAC y RMN.

Resultados: Veintinueve pacientes fueron diagnosticados de PTC entre 1994 y 2004. El 89,6\% (26) de los pacientes fueron mujeres y el $10,4 \%$ (3) fueron varones. La incidencia de PTC en nuestra población oftalmológica fue de 3,2 por 100.000 y de 5,1 por 100000 si se considera la población general dependiente de nuestro hospital.

Conclusión: La incidencia de PTC encontrada fue mayor que la publicada previamente.

Palabras clave: PTC, incidencia, mujer, obesidad.

\begin{abstract}
Objective: To determine the epidemiology of pseudotumor cerebri (PTC) as seen in patients attending our ophthalmology department.

Methods: The medical records of patients diagnosed with PTC in our hospital during a ten-year period (1994 to 2004) were obtained, with only those patients with PTC diagnosed in our ophthalmology department being considered. The following data were reviewed: sex, age at diagnosis, body mass index (BMI), opening cerebrospinal fluid (CSF) pressure, and the results of CT and MRI.

Results: Twenty-nine patients were diagnosed with PTC during this time period. Twenty-six were females $(89.6 \%)$ and 3 were males $(10.4 \%)$. The calculated incidence of PTC in our population was 3.2 per 100.000 persons and 5.1 per 100.000 persons in the general population that attend our hospital.

Conclusion: The incidence of PTC was higher than that previously reported (Arch Soc Esp Oftalmol 2007; 82: 219-222).
\end{abstract}

Key words: PTC, incidence, female, obesity.

\footnotetext{
Recibido: 18/5/05. Aceptado: 26/3/07.

Hospital General. Medina del Campo. Valladolid. España.

1 Doctor en Medicina.

2 Licenciado en Medicina.

3 Doctor en Medicina. Bioestadística.

Correspondencia:

V.M. Asensio Sánchez

Hospital General Servicio Castellano-Leonés de Salud

Servicio de Oftalmología

Medina del Campo (Valladolid)

España

E-mail: vasensio@hmdc.sacyl.es
} 


\section{INTRODUCCIÓN}

El pseudotumor cerebral (PTC) es un proceso neuro-oftalmológico que afecta típicamente a mujeres jóvenes/edad media y obesas. Los criterios diagnósticos son: neuroimágenes normales, presión elevada de salida del líquido cefalorraquídeo (LCR) en la punción lumbar con estudio bioquímico normal, signos y síntomas relacionados con la hipertensión craneal y la no presencia de lesiones neurológicas localizantes, excepto parálisis del sexto par craneal $(1,2)$. Actualmente el término PTC ha sido sustituido por el de hipertensión craneal benigna idiopática o hipertensión craneal idiopática (1). El objetivo de este trabajo es presentar los resultados epidemiológicos de PTC obtenidos en la población dependiente de nuestra área sanitaria y diagnosticados por el servicio de oftalmología.

\section{SUJETO, MATERIAL Y MÉTODOS}

Se revisaron los historiales clínicos de todos los pacientes diagnosticados de PTC por el servicio de oftalmología durante los años 1994 y 2004. Se analizó el motivo de consulta, la edad, sexo, peso, altura, el índice corporal masa (ICM), los resultados de la tomografía axial computerizada (TAC), resonancia magnética nuclear (RMN) y el LCR, su composición y presión de salida. Los pacientes que no tenían estos datos en la historia clínica no fueron incluidos en el estudio.

Definiciones:

— Índice corporal masa (ICM): Índice del peso de una persona en relación con su altura.

- Obesidad: Cuando el ICM es igual o superior a $30 \mathrm{~kg} / \mathrm{m}^{2}$.

- Obesidad grado 2: ICM entre $35-40 \mathrm{~kg} / \mathrm{m}^{2}$.

- Presión de salida del líquido cefalorraquídeo (LCR): Elevada cuando es mayor de $200 \mathrm{~mm} \mathrm{H}_{2} \mathrm{O}$ en personas no obesas y mayor de $250 \mathrm{~mm} \mathrm{H}_{2} \mathrm{O}$ en personas obesas.

\section{RESULTADOS}

Entre los años 1994 y 2004 se diagnosticaron en nuestro hospital 42 pacientes con PTC, de ellos 13 fueron diagnosticados por el servicio de medicina interna, por lo que no han sido considerados en este estudio y 29 fueron diagnosticados por indicación del servicio de oftalmología. Todos los pacientes eran españoles y habían nacido en la zona de influencia del hospital. De los 29 pacientes considerados, 13 (45\%) fueron remitidos a la consulta de oftalmología por cefalea frontal, occipital o en casco asociada a fenómenos vegetativos, dos pacientes (7\%) por amaurosis fugax y en 14 casos (48\%) el paciente no refería ninguna sintomatología (tabla I). El 89,6\% (26) de los pacientes fueron mujeres y el $10,4 \%$ (3) fueron varones. La edad en el momento del diagnóstico era de 34,38 años (rango, 20-55 años). Los 29 pacientes eran obesos, 20 (69\%) con un ICM de $30 \mathrm{~kg} / \mathrm{m}^{2}$ y $9(31 \%)$ con un ICM superior a $35 \mathrm{~kg} / \mathrm{m}^{2}$. Todos tenían una TAC normal, en 10 casos se completó el estudio con RMN sin aportar datos de interés y en un paciente se realizó una RMN medular. La presión de salida del LCR fue de 390 D.E. 64,45 mm H2O (320-470 mm $\left.\mathrm{m}_{2} \mathrm{O}\right)$ con estudio bioquímico normal (tabla II). No se encontró relación entre el grado de obesidad y la presión del LCR. La incidencia calculada de PTC en nuestra población oftalmológica fue de 3,2 por 100.000 y de 5,1 por 100.000 si se considera la población general dependiente de nuestro hospital.

\section{DISCUSIÓN}

El pseudotumor cerebral o hipertensión craneal benigna idiopática es un proceso neuro-oftalmológico de etiología desconocida más frecuente en mujeres obesas entre la segunda y cuarta década de la vida. Se caracteriza por un aumento de la presión cerebral, sin evidencia de una masa intracraneal,

Tabla I. Clínica de presentación PTC

\begin{tabular}{lc}
\hline Pacientes $(\%)$ & Síntoma \\
\hline $13(45 \%)$ & Cefalea inespecífica \\
$2(7 \%)$ & amaurosis fugax \\
$14(48 \%)$ & asintomáticos \\
\hline \hline
\end{tabular}

PTC: Pseudotumor cerebral.

Tabla II. Datos demográficos y clínicos

Sexo: V: 3 M: 26

Edad: 34,38 años (20-55 años)

LCR: 390 D.E. $64,45 \mathrm{mmH}_{2} \mathrm{O}\left(320-470 \mathrm{mmH}_{2} \mathrm{O}\right)$

Obesidad: $100 \%$

V: varón; M: mujer; LCR: líquido cefalorraquídeo. 
aumento de tamaño de los ventrículos o composición anormal del líquido cefalorraquídeo (1-3). El PTC es un proceso clínico de especial importancia en oftalmología, pues a pesar de ser denominado hipertensión craneal benigna idiopática, no es una patología visualmente benigna, ya que se asocia a pérdida de visión lenta, progresiva e irreversible causada por un papiledema prolongado con atrofia óptica secundaria (2-5). En nuestro estudio hasta el $48 \%$ de los pacientes eran asintomáticos, lo cual da más valor al conocimiento de esta enfermedad por parte del oftalmólogo. La incidencia anual de PTC en la población general es de hasta 2/100.000 habitantes (1-2). Kesler et al (6) consideran que la incidencia de PTC en la población israelí oscila entre 0,57 y 0,94 por 100.000 personas. En el estado de Minnesota es de 1 por 100.000 y en Louisiana de 0,9 por 100.000 habitantes (6). En el estudio que se presenta la incidencia es de 3,2 por 100000 en pacientes oftalmológicos y de 5,1 por 100.000 en la población general dependiente de nuestra área de salud, cifra muy superior a la descrita en países occidentales $(2,6)$. Los estudios caso control muestran una gran relación entre PTC y obesidad, que puede estar presente en el $90 \%$ de las personas con PTC $(1,2)$. En este trabajo el $100 \%$ de los pacientes eran obesos y en el $31 \%$ de los casos presentaban una obesidad grado 2 lo cual refleja los malos hábitos higiénico-dietéticos de la zona de influencia de nuestro hospital. Kesler et al (7) describen 141 pacientes con PTC en los que el 77,8\% fueron considerados significativamente obesos. El PTC afecta típicamente a mujeres jóvenes, en nuestro estudio lo eran el $90 \%$ con edades entre 20 y 55 años, datos superponibles a los descritos por Kesler et al (6) con un $93,4 \%$ de mujeres con edad media de 31,61 años. En este estudio todas las punciones lumbares fueron realizadas por la misma persona con especial cuidado en colocar la cabeza del paciente en posición neutra y las piernas extendidas pasivamente después del pinchazo, evitando así cualquier elevación de la presión del LCR secundaria a la postura. En nuestros pacientes la presión de salida del LCR oscilaba entre 320-470 mm H2O (390 DE. 64,45). Kesler et al. (6) en su estudio epidemiológico describen una presión del LCR de 340 DE. 93,99 $\mathrm{mmH}_{2} \mathrm{O}$, comparable a la de nuestros casos. Del estudio que presentamos se deduce que el pseudotunor cerebral es un proceso no infrecuente en la zona de influencia de nuestro hospital, siendo un reflejo del aumento de la obesidad en la población general de nuestro medio y destacando el papel de oftalmólogo en el diagnóstico precoz de una patología con elevada morbilidad ocular.

\section{BIBLIOGRAFÍA}

1. Friedman DI, Jacobson DM. Diagnostic criteria for idiopathic intracranial hypertension. Neurology 2002; 59: 1492-1495.

2. Friedman DI, Jacobson DM. Idiopathic intracranial hypertension. J Neuroophthalmol 2004; 24: 138-145.

3. Silberstein SD, McKinstry RC 3rd. The death of idiopathic intracranial hypertension? Neurology 2003; 60: 14061407.

4. Horgan HC, McFadzean RM, Lee WR. Idiopathic intracranial hypertension: a case report with optic nerve histopathology. J Neuroophthalmol 1999; 19: 182-185.

5. Digre KB. Not so benign intracranial hypertension. BMJ 2003; 326: 613-614.

6. Kesler A, Gadoth N. Epidemiology of idiopathic intracranial hipertensión in Israel. J Neuroophthalmol 2001; 21: 12-14.

7. Kesler A, Goldhammer Y, Gadoth N. Do men with pseudotumor cerebri share the same characteristic as women? A retrospective review of 141 cases. J Neuroophthalmol 2001; 21: 15-17. 